\title{
Binary Code Design for High-Frequency Ultrasound
}

\author{
Sheng-Wen Huang and Pai-Chi Li, Senior Member, IEEE
}

\begin{abstract}
This paper proposes an approach to designing binary codes suitable for high-frequency applications of coded excitation in medical ultrasound. For a highfrequency ultrasound system, transmitting well-designed binary codes with a low sampling ratio (i.e., the bit rate divided by the transducer center frequency) is a practical way to improve the signal-to-noise ratio (SNR) because the challenge of implementing arbitrary-waveform generators for transmitting nonbinary codes increases with the frequency and the switching speed of square-wave pulsers are limited. One conventional approach designs codes using a base sequence that modulates wideband sequences up to the transducer passband. Because a major portion of codes is excluded as a candidate, codes designed using this approach typically need long compression filters for restoring the axial resolution, and they do not improve the SNR efficiently. In contrast, the approach proposed here searches all the codes that match the transducer passband; hence, the resultant codes exhibit better performance. The technique was tested using a bit rate of $50 \mathrm{MHz}$ and a sampling ratio of 2. For a transducer with an ideal Gaussian frequency response with a center frequency of $25 \mathrm{MHz}$ and a $-6 \mathrm{~dB}$ bandwidth of $15 \mathrm{MHz}$, the SNR for the same sidelobe extent was 1 to $6 \mathrm{~dB}$ higher for the codes designed using the proposed approach compared with those designed using the conventional approach. When a real transducer response with a center frequency of $26.4 \mathrm{MHz}$ and a oneway $-6 \mathrm{~dB}$ bandwidth of $20.7 \mathrm{MHz}$ was considered, the codes designed using the proposed approach were superior by 0.5 to $5 \mathrm{~dB}$. Therefore, our approach is better than the conventional approach for designing binary codes for highfrequency ultrasound, with the results indicating that the moderate bit rate of $50 \mathrm{MHz}$ will suffice when the ultrasonic center frequency is $25 \mathrm{MHz}$.
\end{abstract}

\section{INTRODUCTION}

$\mathrm{A}$ PRIMARY application of coded excitation in medical ultrasound is improving the signal-to-noise ratio (SNR) without increasing the peak acoustic power [1], and the papers on coded waveforms (e.g., [2], [3]) published in this journal describe some of the recent progress and offer extensive references to previous studies in diverse applications. Fig. 1 shows a block diagram of a coded excitation system, in which each channel has a transmitter able to transmit the desired code (which has a wide bandwidth

Manuscript received February 23, 2006; accepted December 28, 2006. Partial support from the National Science Council of R.O.C. under Grant NSC 95-2218-E-002-036 and from the National Health Research Institutes of R.O.C. is gratefully acknowledged.

The authors are with the Department of Electrical Engineering, National Taiwan University, Taipei, Taiwan (e-mail: paichi@cc.ee.ntu.edu.tw).

Digital Object Identifier 10.1109/TUFFC.2007.340

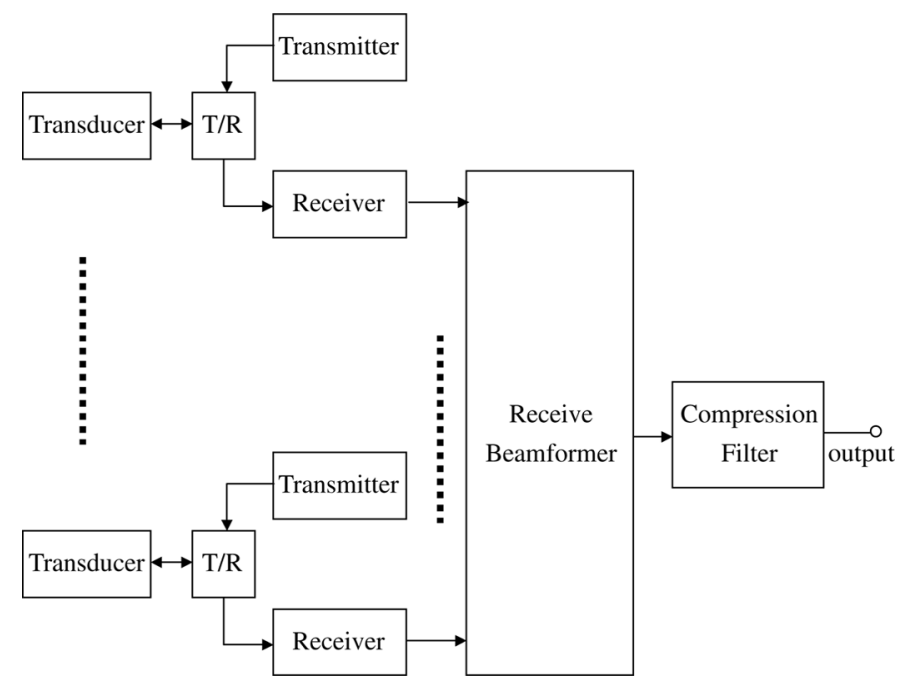

Fig. 1. Block diagram of a coded excitation system.

and an increased pulse length), and a compression filter whose coefficients and length are strongly influenced by the code is placed after the receive beamformer so as to restore the axial resolution. The performance of pulse compression is generally characterized by the mainlobe width (related to the axial resolution) and the sidelobe level (related to the dynamic range and contrast resolution) of the compressed pulse, and the SNR improvement (i.e., the difference between the SNRs after and before pulse compression).

Codes can be classified according to the required transmitter type. A nonbinary code necessitates an arbitrarywaveform transmitter, and a binary code can be transmitted with a square-wave pulser. Although it is easy to design nonbinary codes with good compression performances, such codes have not been widely used in array systems mainly due to the increased hardware complexity and cost associated with the requirement for multiple arbitrary-waveform transmitters. Furthermore, the challenge of implementing an arbitrary-waveform transmitter increases with the center frequency of the transducer (or the code), and it may still be impractical to use codes other than binary ones for a high-frequency (e.g., $25 \mathrm{MHz}$ ) ultrasound system that images using an array of transducers. The difficulty of designing binary codes also increases with frequency due to the limited switching speed of squarewave pulsers and, consequently, the allowable sampling ratio decreases as the center frequency of the transducer increases (where the sampling ratio is the bit rate divided by the transducer center frequency). 
In this paper, we focus on designing single-firing (or single-transmit) binary codes having a low sampling ratio (e.g., 2) and efficiently improving the SNR. Here a code improving SNR efficiently means that it provides a high ratio of SNR improvement to sidelobe duration after pulse compression. Given a certain code length (in units of time), using a lower sampling ratio is equivalent to having fewer tunable bits and fewer degrees of freedom in the code design, which results in worse performance. Note that in order to cover the full bandwidth of the transducer, the sampling ratio is generally at least 2 .

In medical ultrasound, a typical approach to generating a binary radio-frequency (RF) code with a low sampling ratio is modulating an $N_{\mathrm{C}}$-bit wideband sequence $c_{\mathrm{WB}}(n)$, $n=0,1, \ldots, N_{\mathrm{C}}-1$, up to the transducer band using an $N_{\mathrm{B}}$-bit base sequence $b(n), n=0,1, \ldots, N_{\mathrm{B}}-1$. Zeros are inserted into $c_{\mathrm{WB}}(n)$ to get:

$$
\begin{aligned}
& c_{\mathrm{WB}}^{\prime}(n)= \\
& \begin{cases}c_{\mathrm{WB}}(m) & \text { if } n=N_{\mathrm{B}} m \text { for } m=0,1, \ldots, N_{\mathrm{C}}-1, \\
0 & \text { elsewhere }\end{cases}
\end{aligned}
$$

and then $c_{\mathrm{WB}}^{\prime}(n)$ is convolved with $b(n)$ to form the final $N$-bit RF code $c_{\mathrm{RF}}(n), n=0,1, \ldots, N-1$, with $N=$ $N_{\mathrm{C}} N_{\mathrm{B}}$ [2]-[4]. Explicitly:

$$
\begin{gathered}
c_{\mathrm{RF}}\left(p+q N_{\mathrm{B}}\right)=b(p) c_{\mathrm{WB}}(q), \\
p=0,1, \ldots, N_{\mathrm{B}}-1, \\
q=0,1, \ldots, N_{\mathrm{C}}-1 .
\end{gathered}
$$

The wideband sequence is chosen to have the desired properties such as enabling range sidelobe cancellation [2] or benefiting inverse filtering [3], [4], and the base sequence (together with the bit rate) is used to control the band of the RF code [2]-[4]. We call such an approach the modulation approach. Because it has at most $\left[\left(N_{\mathrm{C}}-1\right)+\left(N_{\mathrm{B}}-1\right)\right]$ adjustable bits, a major portion of $N$-bit codes is excluded as a candidate. This means that some codes with good compression performances are not considered in order to reduce the design effort. Therefore, not all single-firing codes designed using the approach provide a high SNR improvement together with a short sidelobe duration and a short near-field dead zone.

Although the exhaustive-search approach (checking all $N$-bit codes in order to find the best RF code) is unfeasible in general, there are feasible approaches that are better than the modulation approach. In this study, we developed an indicator-based, random-search approach to finding codes with good compression performance based on a genetic algorithm [5]. This indicator is easy to calculate, and a code with a high-indicator value usually has good compression performance. Hence, the adoption of the indicator makes the proposed approach feasible. As shown in Section IV, the compression performances of codes designed using this approach are superior to those designed using the modulation approach.

This paper is organized as follows. Section II describes the criterion for evaluating the codes that are used to im- prove the SNR. Section III details the indicator-based, random-search approach to binary code design. The genetic algorithm used for the code search also is presented. Section IV demonstrates the efficacy of the proposed approach and discusses its characteristics, and the paper concludes in Section V.

\section{Criterion for Evaluating Codes}

For single-firing codes, restoring the axial resolution by compressing the pulses is achieved by filtering. In order to clarify the criterion for evaluating codes, we must first describe how to design the compression filter used in this study.

\section{A. Design of the Compression Filter}

In general, a time-domain compression filter is preferable to a frequency-domain filter because the latter is vulnerable to the presence of incomplete echoes in the near field. In this study, we adopted a previously proposed method [6] providing direct control over the sidelobe level to design time-domain compression filters at baseband. If $h_{\mathrm{t}}(t)$ is the one-way impulse response of the transducer, the digitized echo signal corresponding to an $N$-bit code $c_{\mathrm{RF}}(n)$ from a point target can be modeled as:

$$
e(n)=\left[c_{\mathrm{RF}}(t) \otimes h_{\mathrm{t}}(t) \otimes h_{\mathrm{t}}(t)\right]_{t=n / f_{\mathrm{s}}},
$$

where $\otimes$ denotes convolution,

$$
c_{\mathrm{RF}}(t)=\sum_{n=0}^{N-1} c_{\mathrm{RF}}(n) \Pi\left(f_{\mathrm{b}} t-n+\frac{N}{2}\right),
$$

$f_{\mathrm{b}}$ is the bit rate, $f_{\mathrm{s}}$ is the sampling rate of the analog-todigital converter $(\mathrm{ADC})$ at the receiver, and $\Pi(t)=1$ if $0 \leq t \leq 1$ and 0 elsewhere. The $e(n)$ is demodulated into a baseband signal:

$$
e_{\mathrm{b}}(n)=h_{\mathrm{L}}(n) \otimes\left[e(n) \cdot \exp \left(-j 2 \pi f_{0} n / f_{\mathrm{s}}\right)\right],
$$

where $f_{0}$ is the center frequency of the transducer and $h_{\mathrm{L}}(n)$ is a low-pass filter. The $e_{\mathrm{b}}[n]$ then is downsampled by a factor of $D$ to obtain $e_{\mathrm{d}}(n)=e_{\mathrm{b}}(D n)$. Filtering $e_{\mathrm{d}}(n)$ with a compression filter $h_{\mathrm{o}}(n)$ restores the axial resolution in the output signal.

Given a filter length $N_{\mathrm{f}}, h_{\mathrm{o}}(n)$ is designed to maximize the output SNR under the constraints on the mainlobe width and the sidelobe level. Let $m$ denote the index of the peak position of $e_{\mathrm{d}, \mathrm{c}}(n), I_{\mathrm{sl}}$ denote the index set of the sidelobes, $s$ specify the allowable peak sidelobe level, and $s_{\mathrm{dB}}=20 \log s$, then explicitly:

$$
\begin{aligned}
& h_{\mathrm{o}}(n)=\arg \min _{h(n)} \sigma_{n_{\mathrm{d}, \mathrm{c}}}^{2}(0) \text { subject to } \\
& \left\{\begin{array}{c}
e_{\mathrm{d}, \mathrm{c}}(m)=1 \\
\left|\operatorname{Re}\left\{e_{\mathrm{d}, \mathrm{c}}(n)\right\}\right| \leq s / \sqrt{2} \text { for } n \in I_{\mathrm{sl}}, \\
\left|\operatorname{Im}\left\{e_{\mathrm{d}, \mathrm{c}}(n)\right\}\right| \leq s / \sqrt{2} \text { for } n \in I_{\mathrm{sl}}
\end{array}\right.
\end{aligned}
$$


where $\sigma_{n_{\mathrm{d}, \mathrm{c}}}^{2}$ denotes the power of the thermal noise $n_{\mathrm{d}, \mathrm{c}}(n)$ after compression, $e_{\mathrm{d}, \mathrm{c}}(n)=e_{\mathrm{d}}(n) \otimes h(n), h(n)$ has a length of $N_{\mathrm{f}}$, and $\operatorname{Re}\{\cdot\}$ and $\operatorname{Im}\{\cdot\}$ denote the real and imaginary parts, respectively. The details for solving (6) are available elsewhere [6].

\section{B. Definition of Code Efficiency}

The near-field dead zone of a coded excitation system using a code-filter combination $\left\{c_{\mathrm{RF}}(n ; N), h_{\mathrm{o}}\left(n ; N_{\mathrm{f}}\right)\right\}$ is $\left(T_{\mathrm{c}}+T_{\mathrm{f}}\right) / 2$ larger than that of a pulsed excitation system, where $T_{\mathrm{c}}=N / f_{\mathrm{b}}$ and $T_{\mathrm{f}}=N_{\mathrm{f}} D / f_{\mathrm{s}}$ (both in units of time) are the code length and the filter length, respectively. In addition, the sidelobe of a point target spans a duration of $\left(T_{\mathrm{c}}+T_{\mathrm{f}}\right)$ for this coded excitation system. Therefore, the value of $\left(T_{\mathrm{c}}+T_{\mathrm{f}}\right)$ should be as small as possible, and a given code-filter combination is better than another if it provides the same SNR improvement and has a lower $\left(T_{\mathrm{c}}+T_{\mathrm{f}}\right)$. Because the output SNR ideally should be proportional to both $T_{\mathrm{c}}$ and $\left(T_{\mathrm{c}}+T_{\mathrm{f}}\right)$, it is reasonable to treat a codefilter combination as better if it has a higher code-filter efficiency:

$$
\begin{aligned}
& E_{\mathrm{cf}}\left[c_{\mathrm{RF}}(n ; N), h_{\mathrm{o}}\left(n ; N_{\mathrm{f}}\right)\right] \equiv \\
& \operatorname{SNR}_{\mathrm{dB}}-10 \log _{10}\left[\left(T_{\mathrm{c}}+T_{\mathrm{f}}\right) /\left(T_{0} / 2\right)\right],
\end{aligned}
$$

where $\mathrm{SNR}_{\mathrm{dB}}$ is the output SNR in decibels, and $T_{0}=f_{0}^{-1}$. We can use (7) to fairly compare two code-filter combinations even if they provide different SNR improvements.

Note that given a code $c_{\mathrm{RF}}(n ; N)$, there is a one-to-one correspondence between $N_{\mathrm{f}}$ and $h_{\mathrm{o}}\left(n ; N_{\mathrm{f}}\right)$. That is:

$$
E_{\mathrm{cf}}\left[c_{\mathrm{RF}}(n ; N), h_{\mathrm{o}}\left(n ; N_{\mathrm{f}}\right)\right]=E_{\mathrm{cf}}\left[c_{\mathrm{RF}}(n ; N), N_{\mathrm{f}}\right],
$$

and there exists an $N_{\mathrm{f}}$ (or a $T_{\mathrm{f}}$ ) that results in the maximal $E_{\mathrm{cf}}$ value for the code. Defining the efficiency $E_{\mathrm{c}}$ of a code $c_{\mathrm{RF}}(n ; N)$ as:

$$
E_{\mathrm{c}}\left[c_{\mathrm{RF}}(n ; N)\right] \equiv \max _{N_{\mathrm{f}}}\left\{E_{\mathrm{cf}}\left[c_{\mathrm{RF}}(n ; N), N_{\mathrm{f}}\right]\right\},
$$

means that a code which is easier to compress and/or improves the SNR to a greater extent will have a higher $E_{\mathrm{c}}$ value. Therefore, in this study a code is treated as more efficient at improving the SNR or having better compression performance if its $E_{\mathrm{c}}$ value is higher.

\section{The Indicator-Based Search Approach to Binary Code Design}

Genetic algorithms may be used to stochastically search a domain that is too large to be searched exhaustively [5]. A genetic algorithm previously was used to design binary codes suitable for being compressed with inverse filtering in the frequency domain [7]. In the present study, we developed a genetic algorithm to design binary codes with high efficiencies. Because given a code, for obtaining its efficiency, typically tens of compression filters corresponding

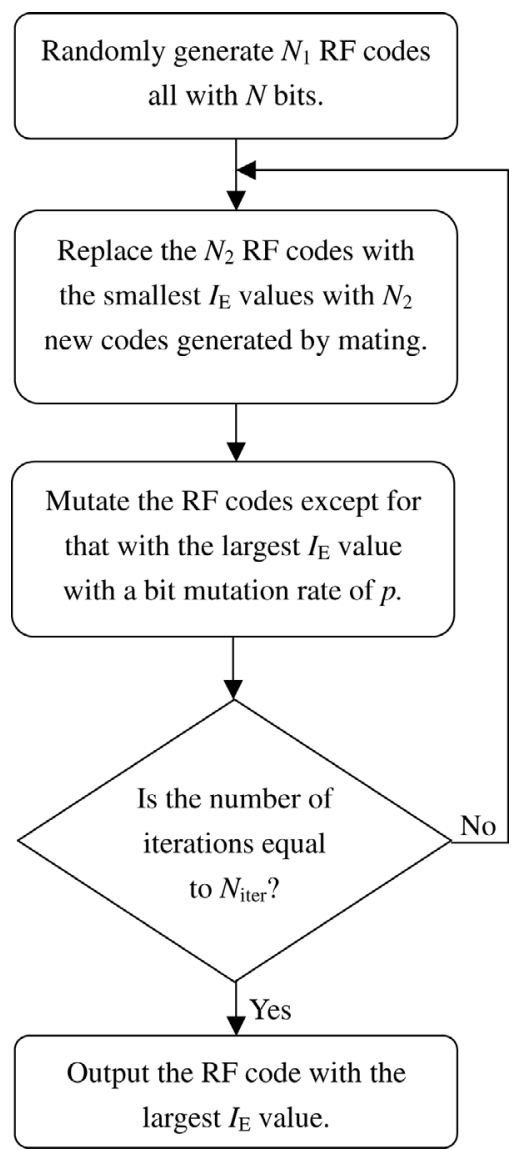

Fig. 2. Flow diagram of the genetic algorithm used in the proposed search approach.

to different $N_{\mathrm{f}}$ 's need to be designed [see (9)] and designing a compression filter means solving a quadratic programming problem [see (6)] [6], it is evident that directly calculating the efficiency of each code during the search process is computationally expensive. Therefore, in our algorithm the following indicator that is easier to calculate while being closely correlated with the code efficiency was used as the parameter to be optimized during the search process:

$$
I_{\mathrm{E}}\left[c_{\mathrm{RF}}(n ; N)\right] \equiv\left\|\left\{\frac{R_{c_{\mathrm{RF}}}(t)}{R_{c_{\mathrm{RF}}}(0)}-\frac{R_{b}(t)}{R_{b}(0)}\right\} \otimes h_{\mathrm{t}}(t)\right\|^{-1},
$$

where $R_{a}(t)=a(t) \otimes a^{*}(-t)$ denotes the autocorrelation function of the signal $a(t)$ [representing $c_{\mathrm{RF}}(t)$ or $b(t)$ here],

$$
b(t)=\sum_{n=0}^{N_{\mathrm{B}}-1} b(n) \Pi\left(f_{\mathrm{b}} t-n+\frac{N_{\mathrm{B}}}{2}\right),
$$

$R_{c_{\mathrm{RF}}}(0)=\left(N / f_{\mathrm{b}}\right)$, and $R_{b}(0)=\left(N_{\mathrm{B}} / f_{\mathrm{b}}\right)$. If the spectrum of an $\mathrm{RF}$ code in the transducer band is similar to that of the base sequence (in terms of the normalized amplitude), it will have a large $I_{\mathrm{E}}$ value. Because, in general, the base sequence (such as $\{1,-1\}$ or $\{1,-1,1,-1\}$ ) has a smooth spectrum, RF codes with large $I_{\mathrm{E}}$ values tend to have a smooth spectrum. Therefore, they are easy to compress 
TABLE I

Hexadecimal Representations of the RF Codes Designed Using the Search Approach (Second Column) and the Modulation Approach (Fifth Column) For $b(n)=(-1)^{n}$ and $N_{\mathrm{B}}=4 .^{1}$

\begin{tabular}{ccccc}
\hline & \multicolumn{3}{c}{ Search approach } & \multicolumn{3}{c}{ Modulation approach } \\
\cline { 2 - 5 }$N$ & RF code & $N_{\mathrm{C}}$ & Wideband \\
sequence & RF code \\
\hline 32 & 252A5551 & 8 & $1 \mathrm{~A}$ & 555AA5A5 \\
36 & 75558AD4B & 9 & 068 & 55AA5A555 \\
40 & 4AD52AAAFB & 10 & $0 \mathrm{D} 0$ & 55AA5A5555 \\
44 & 5D554BA9529 & 11 & 065 & 5555AA55A5A \\
48 & 692952A15555 & 12 & $0 \mathrm{CA}$ & 5555AA55A5A5 \\
52 & 755552A952969 & 13 & $00 \mathrm{CA}$ & 55555AA55A5A5 \\
56 & 755556AB54A5A1 & 14 & 0195 & 55555AA55A5A A \\
60 & 7555542A956A5AD & 15 & 0EED & 555AAA5AAA5AA5A \\
64 & 2D695AA550AAAABB & 16 & 2833 & 55A5A55555AA55AA \\
\hline
\end{tabular}

\footnotetext{
${ }^{1}$ The wideband sequences [9] necessary for the modulation approach are listed in the fourth column. The transducer was assumed to have a Gaussian frequency response with a $60 \%$ fractional bandwidth, and the additive noise sampled by the ADC was assumed to be white.
}

and exhibit a high efficiency. That is, the base sequence is used to control the spectrum shape of the RF code in the proposed search approach, as is also the case for the modulation approach.

Fig. 2 shows the flow diagram of the genetic algorithm for RF code design. In the second step, the probability of a code being chosen as a parent code for mating is proportional to its $I_{\mathrm{E}}$ value. A pair of parent codes $\left\{c_{\mathrm{RF}, 1}(n), c_{\mathrm{RF}, 2}(n)\right\}$ generates two offspring codes $c_{\mathrm{RF}, 1}^{\prime}(n)$ and $c_{\mathrm{RF}, 2}^{\prime}(n)$ according to the following rule:

$$
c_{\mathrm{RF}, k}^{\prime}(n)=\left\{\begin{array}{ll}
c_{\mathrm{RF}, k}(n) & \text { if } u(n) \geq 0.5 \\
c_{\mathrm{RF}, 3-k}(n) & \text { otherwise }
\end{array},\right.
$$

where $u(n), n=0,1, \ldots, N-1$, are random numbers generated with a uniform probability density within $[0,1]$, and $k$ equals to 1 or 2 .

\section{Results And Discussion}

A bit rate of $50 \mathrm{MHz}$ was used in this study, except where stated otherwise. Given a fixed bit rate, the efficiency of a code relative to other codes is dominated by the transducer response and the noise characteristics, and it is less dependent on the parameters related to the design of the compression filter, including those of the filter $h_{\mathrm{L}}(n)$, the sampling rate of the $\mathrm{ADC}\left(f_{\mathrm{s}}=200 \mathrm{MHz}\right)$, the downsampling ratio $(D=5)$, the mainlobe width (defined as the central nine points of the compressed signal), and the allowable peak sidelobe level $\left(s=0.01\right.$ or $\left.s_{\mathrm{dB}}=-40 \mathrm{~dB}\right)$. Here $h_{\mathrm{L}}(n)$ was a Kaiser-windowed finite impulse response low-pass filter with filter length $(M+1)=19$, shape parameter $\beta=3.7$, and $-6 \mathrm{~dB}$ cutoff frequency $f_{\mathrm{c}}=0.1125$ $[6],[8]$.

We designed RF codes using the search approach described in Section III and compared their efficiencies with those of the codes designed using the modulation approach. The additive noise sampled by the ADC was assumed to be a white noise. Furthermore, we assumed that the SNR defined as the ratio of the peak signal power to the average noise power at baseband was 1 when transmitting a single-bit signal. Section IV-A describes the RF codes corresponding to an assumed transducer response. Section IV-B presents the impulse response of a transducer and the consequent designs of the matching RF codes. The effects of quantizing filter coefficients on compression performance are discussed in Section IV-C.

\section{A. Results for a Gaussian-Shaped Transducer Response}

Codes were designed assuming that the transducer has a Gaussian frequency response with a center frequency $f_{0}$ of $25 \mathrm{MHz}$ and a $-6 \mathrm{~dB}$ bandwidth of $15 \mathrm{MHz}$. The parameters of the genetic algorithm were $N_{1}=200, N_{2}=100$, $p=1 / N$, and $N_{\text {iter }}=1000$. Given a specific code length and a base sequence, we realized $N_{3}=200$ trials and calculated the efficiencies of the $10 \mathrm{RF}$ codes with the largest $I_{\mathrm{E}}$ values, in which the code with the highest efficiency was chosen to be the final RF code. Twenty-six codes were designed in this way, nine of which correspond to $N=32$ to 64 with a step size of 4 using a two-cycle square wave [i.e., $b(n)=(-1)^{n}$ and $N_{\mathrm{B}}=4$ ] as the base sequence (Table I), with the others corresponding to $N=32$ to 64 with a step size of 2 using a one-cycle square wave [i.e., $b(n)=(-1)^{n}$ and $\left.N_{\mathrm{B}}=2\right]$ as the base sequence (Table II). Note that, before transforming a code into its hexadecimal representation, all the bits equal to -1 were replaced with 0 . Tables I and II also list the codes designed using the modulation approach, which were used as the references. The wideband sequences for the modulation approach were the sequences minimizing the noise enhancement factor [9]. That is, the output SNR will be well maintained if inverse filtering is applied to compress such a sequence (or code) and the noise is white. Note that the above statement neglects the transducer frequency response; a base sequence is necessary for pulse shaping (i.e., controlling the band of the RF code) if the transducer is taken into account. $\mathrm{RF}$ codes have been designed [3], [4] using the modulation approach with previously listed sequences [9]. 
TABLE II

Hexadecimal Representations of the RF Codes Designed Using the Search Approach (Second Column) and the Modulation Approach (Fifth Column) For $b(n)=(-1)^{n}$ and $N_{\mathrm{B}}=2 .{ }^{1}$

\begin{tabular}{ccccc}
\hline & \multicolumn{2}{c}{ Search approach } & \multicolumn{3}{c}{ Modulation approach } \\
\cline { 2 - 5 }$N$ & RF code & $N_{\mathrm{C}}$ & sequence & RF code \\
\hline 32 & 3AAB5A4C & 16 & 2833 & 59955A5A \\
34 & 03AAB5A4C & 17 & 04C2B & 165A5599A \\
36 & 3C96A5577 & 18 & 0B477 & 59A656A6A \\
38 & 1A56AA9B0C & 19 & 17421 & 166A655956 \\
40 & 25AD72AA0C & 20 & 05D39 & 5566A65A96 \\
42 & 1D755AB4F6C & 21 & 07C452 & 16AA5656659 \\
44 & 02494A95457 & 22 & 0F88A4 & 5AA95959965 \\
46 & 0906B5AAAF04 & 23 & 00E6D5 & 1555A969A666 \\
48 & 0E952AEADA4F & 24 & 111E2D & 565656A959A6 \\
50 & 0C17455AA525B & 25 & 073F536 & 16A5AAA665A69 \\
52 & 6214A5556C24F & 26 & 063F56D & 5695AAA6669A6 \\
54 & 0DED2B562ABA6C & 27 & 0F1112D & 15AA56565659A6 \\
56 & 65DD5252A8567B & 28 & 38FEA4D & 5A95AAA99965A6 \\
58 & 07641556AB4A53C & 29 & 031FD5B2 & 15A56AAA6669A59 \\
60 & 6964AD6AAAF04C0 & 30 & 09BF8EB5 & 5969AAA95A99A66 \\
62 & 032CAAA456A521CC & 31 & 1CEADBF0 & 16A5A999A69AAA55 \\
64 & 06B56AD352EAAE80 & 32 & 00F2D533 & 5555AA59A6665A5A \\
\hline
\end{tabular}

${ }^{1}$ The wideband sequences [9] necessary for the modulation approach are listed in the fourth column. The transducer was assumed to have a Gaussian frequency response with a $60 \%$ fractional bandwidth, and the additive noise sampled by the ADC was assumed to be white.

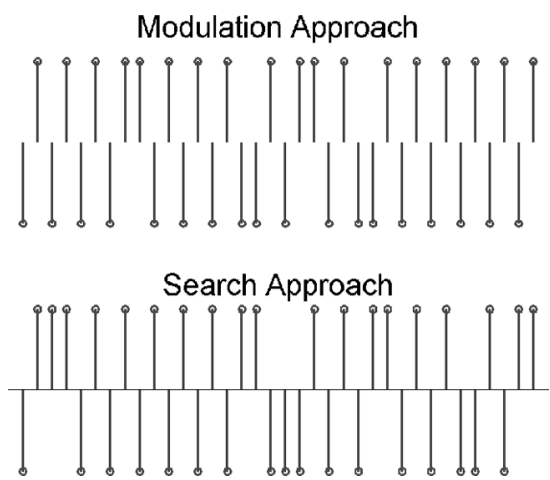

(a)

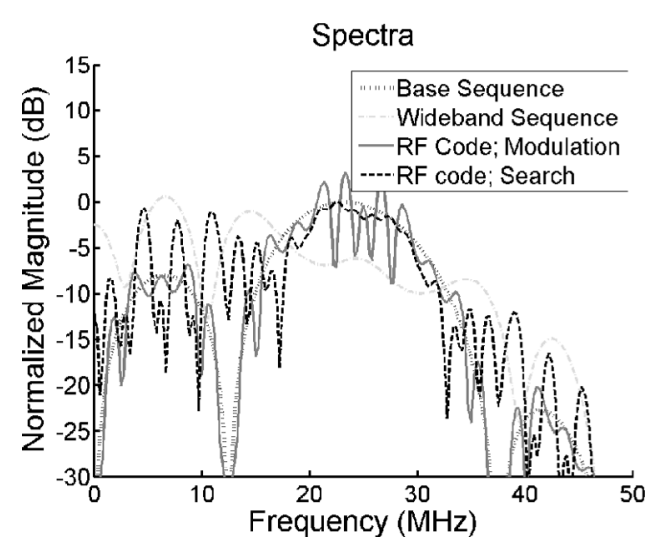

(b)

Fig. 3. (a) 36-bit RF codes designed using the modulation and search approaches using a two-cycle square wave as the base sequence. (b) The normalized spectra of the base sequence, the wideband sequence for the modulation approach, and the RF codes.

As an example, the results from designing a 36-bit $\mathrm{RF}$ code using the modulation and search approaches with the two-cycle base sequence (i.e., $\{1,-1,1,-1\}$ ) are shown in Fig. 3. Fig. 3(a) shows the RF codes designed using the two approaches, and Fig. 3(b) shows the normalized spectra of the base sequence, the wideband sequence for the modulation approach, and the RF codes. The spectrum in the transducer passband of the RF code designed using the search approach is smoother and thus easier to equalize than that designed using the modulation approach, resulting in a better compression performance and a higher efficiency. Note that Fig. 3(b) also illustrates that the base sequence controls the sampling ratio. If the base sequence $\{1,1,-1,-1,1,1,-1,-1\}$ is used instead of $\{1,-1,1,-1\}$, the sampling ratio will be 4 instead of 2 .
Given a code length $T_{\mathrm{c}}, N$ will increase by a factor of 2. Because there are more adjustable bits, the code designed using the search approach will have a higher efficiency traded with a higher bit rate. For example, the 72-bit code "1CE73988CCCCCDD936" operating at a bit rate of $100 \mathrm{MHz}$ has an efficiency of $1.11 \mathrm{~dB}$ (resulting from an SNR of $21.31 \mathrm{~dB}$ at a filter length of $1.375 \mu \mathrm{s}$ ), which is $0.81 \mathrm{~dB}$ higher than that of its corresponding 36 -bit code operating at $50 \mathrm{MHz}$.

Fig. 4(a) shows the efficiencies of codes as functions of their lengths. Each code designed using the search approach has a higher efficiency than its corresponding code designed using the modulation approach with the same base sequence, with the difference ranging between 1 and $6 \mathrm{~dB}$. In addition, the efficiencies of codes designed using 


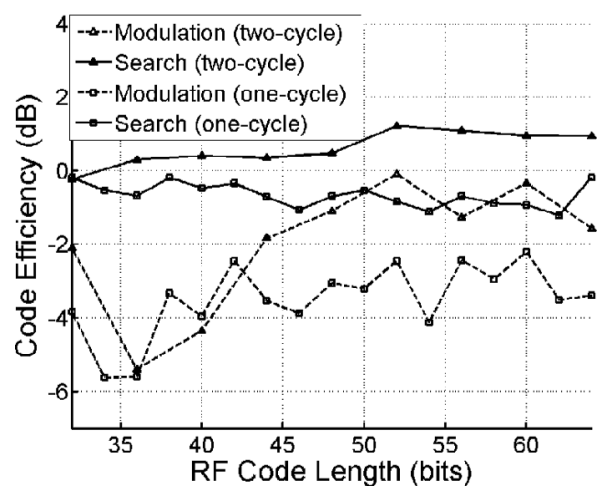

(a)

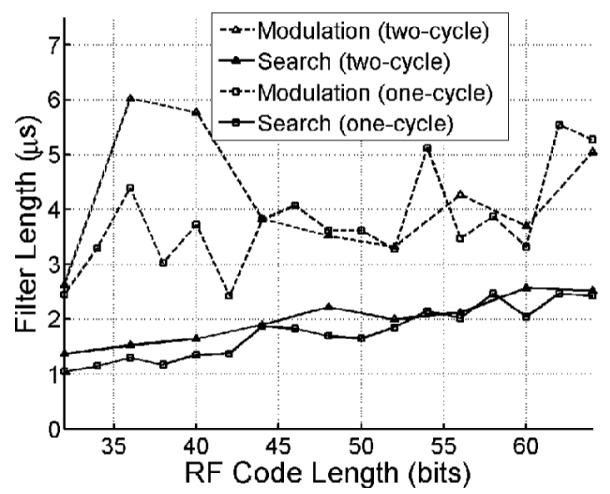

(b)

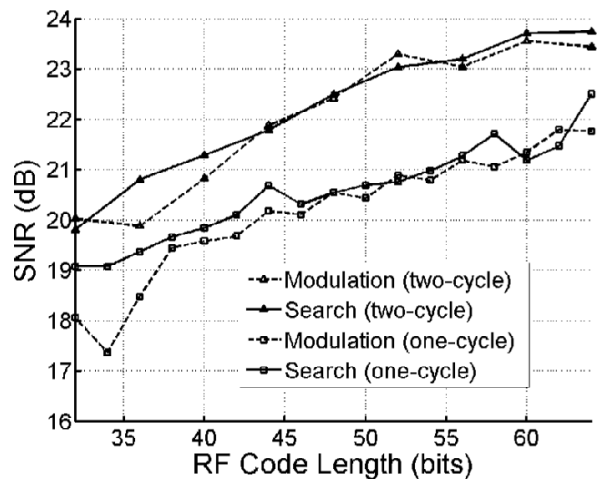

(c)

Fig. 4. Results for a Gaussian-shaped transducer response with a center frequency of $25 \mathrm{MHz}$ and a $-6 \mathrm{~dB}$ bandwidth of $15 \mathrm{MHz}$. (a) The efficiencies of codes as functions of their lengths. (b) The filter lengths that resulted in the maximal $E_{\mathrm{cf}}$ values equal to the code efficiencies. (c) The output SNRs corresponding to the filter lengths shown in (b).

the search approach are more independent of their lengths. Fig. 4(b) shows the filter lengths that resulted in the maximal $E_{\mathrm{cf}}$ values (i.e., the code efficiencies); the filter length is roughly proportional to the code length for the search approach. The ratios of the filter length to the code length range over $[1.9,2.3]$ and $[1.5,2.1]$ with the two- and onecycle base sequences, respectively. In contrast, the filter length fluctuates markedly as the code length increases for the modulation approach; the ratios of the filter length to the code length range over $[3.1,8.4]$ and $[2.8,6.1]$ with the two- and one-cycle base sequences, respectively. Fig. 4(c) shows the output SNRs corresponding to the filter lengths

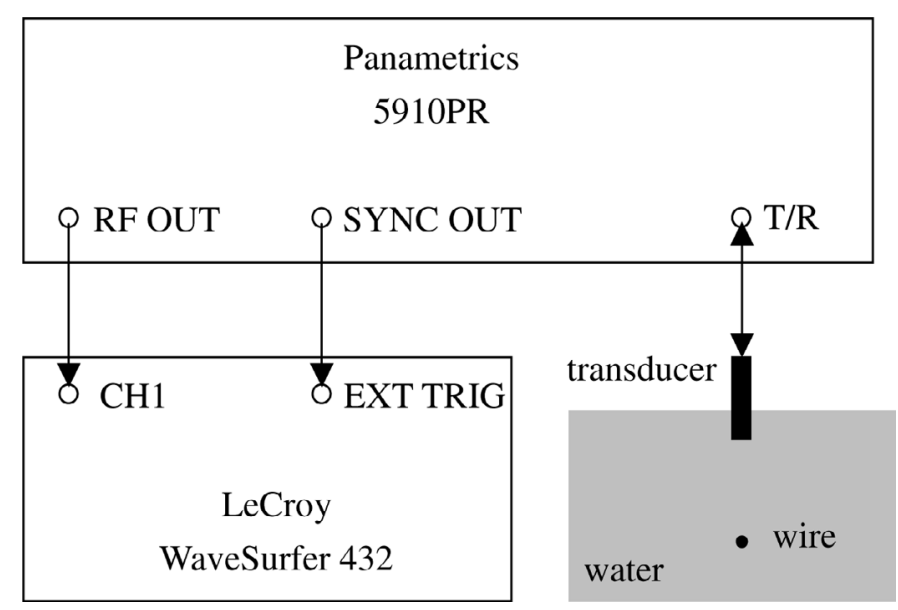

Fig. 5. Block diagram of the experimental setup used to measure the impulse response of a real transducer.

that resulted in the maximal $E_{\mathrm{cf}}$ values. Because the search approach tends to maximize the code efficiency instead of the SNR, some codes designed using this approach have SNRs that are lower than those of the corresponding codes designed using the modulation approach. In general, for a given code length, the SNR differs little between the codes designed using the two approaches; that is, the improvement in code efficiency using the search approach is mainly attributable to the reduction in the filter length. Furthermore, using a two-cycle square wave instead of a one-cycle square wave as the base sequence results in higher SNRs [Fig. 4(c)] due to the better match between the spectrum of the former base sequence and the transducer frequency response. This explains why the codes designed using the two-cycle base sequence have higher efficiencies, as shown in Fig. 4(a).

\section{B. Results for a Measured Transducer Response}

Fig. 5 shows the experimental setup used to measure the impulse response of a real transducer, which had a diameter of $6.35 \mathrm{~mm}$ and a focal length of $12.7 \mathrm{~mm}$ (V324, Panametrics, Waltham, MA). A pulser/receiver (5910PR, Panametrics) operating in pulse-echo mode was used to drive the transducer with a negative impulse, to receive and provide a noninverting $66 \mathrm{~dB}$ gain with $-3 \mathrm{~dB}$ bandwidth between 5 and $400 \mathrm{MHz}$ to the echo signal from a metal wire, and to synchronize an oscilloscope (WaveSurfer 432, LeCroy, Chestnut Ridge, NY) at a pulse repetition frequency of $200 \mathrm{~Hz}$. The wire was measured to have a diameter of $32 \mu \mathrm{m}$ and was placed $12.4 \mathrm{~mm}$ from the transducer. The amplified echo was digitized by the oscilloscope at a sampling rate of $2 \mathrm{GHz}$ with an amplitude resolution of 8 bits.

Assuming that the effects of the finite bandwidths of the transmitted impulse and the amplifier in the receiver were negligible - although a thin wire was used instead of a small sphere - we treated the minus of the recorded echo $e_{\text {pulse }}$ as a scaled approximation of the two-way impulse response of the transducer near the focus. Therefore, the 


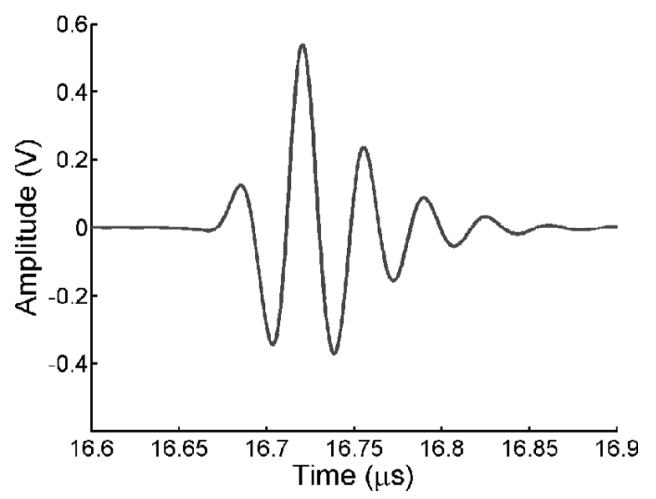

(a)

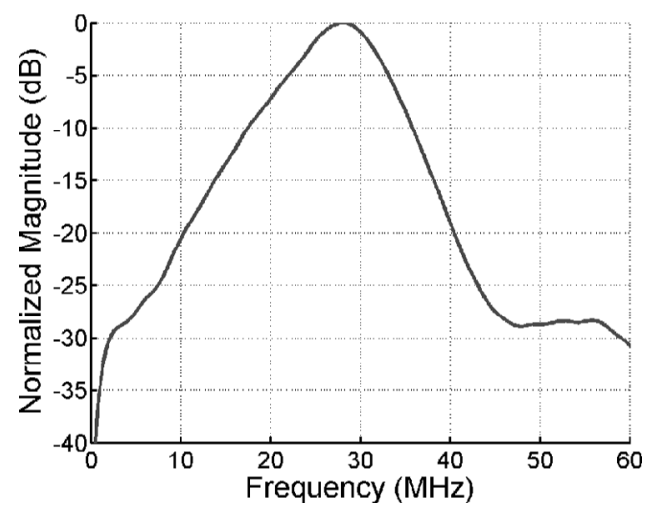

(b)

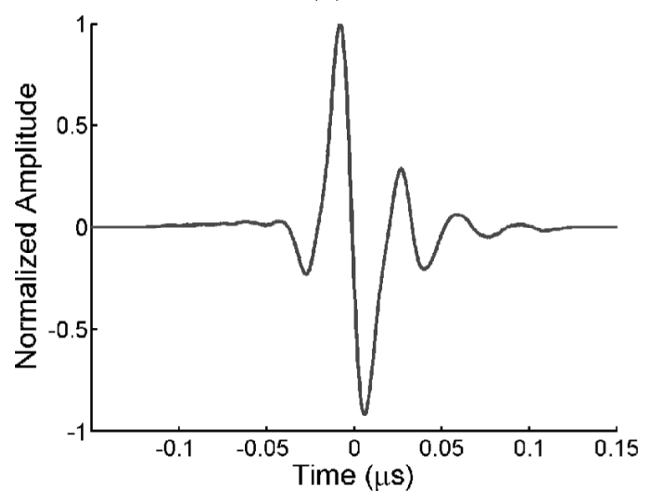

(c)

Fig. 6. (a) The averaged echo signal reflected from a wire. (b) The spectrum of the echo signal. (c) The one-way impulse response derived from the echo signal.

normalized transducer response can be estimated using the following relation: $h_{\mathrm{t}}(t) \propto \mathbf{F}^{-1}\left\lfloor\left(\mathbf{F}\left[-e_{\text {pulse }}(t)\right]\right)^{1 / 2}\right\rfloor$, where $\mathbf{F}$ and $\mathbf{F}^{-1}$ denote forward and inverse Fourier transforms, respectively.

Figs. 6(a) and (b) show the echo signal $e_{\text {pulse }}$ from the wire (which is the average of 2000 records) and its spectrum, respectively. The estimated center frequency and one-way $-6 \mathrm{~dB}$ bandwidth were 26.4 and $20.7 \mathrm{MHz}$, respectively. The estimated transducer impulse response is shown in Fig. 6(c).

Twenty-six RF codes corresponding to the measured transducer response were designed using the search approach with the same parameters as used in Section IVA for the genetic algorithm, nine of which correspond to

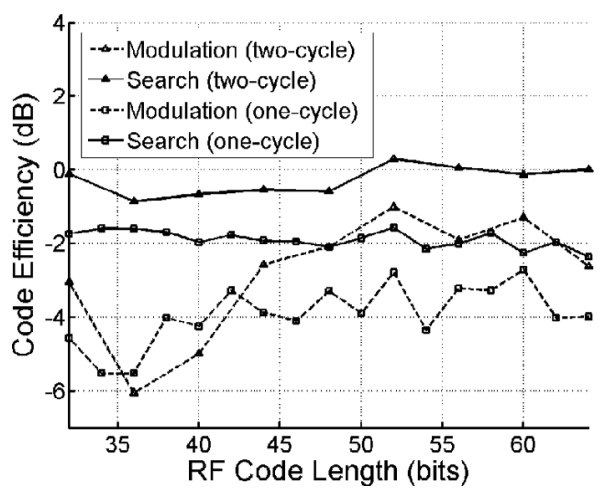

(a)

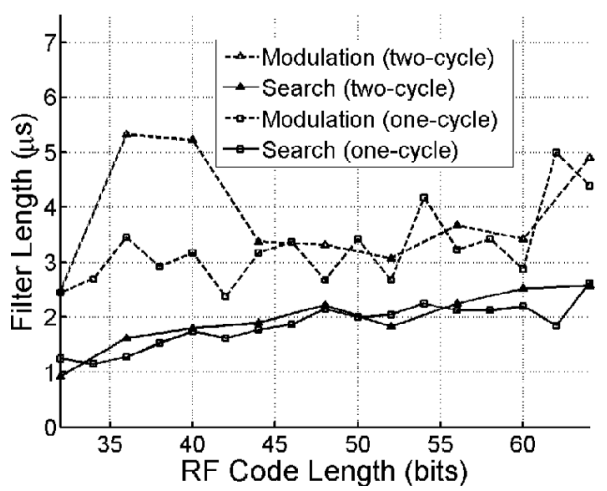

(b)

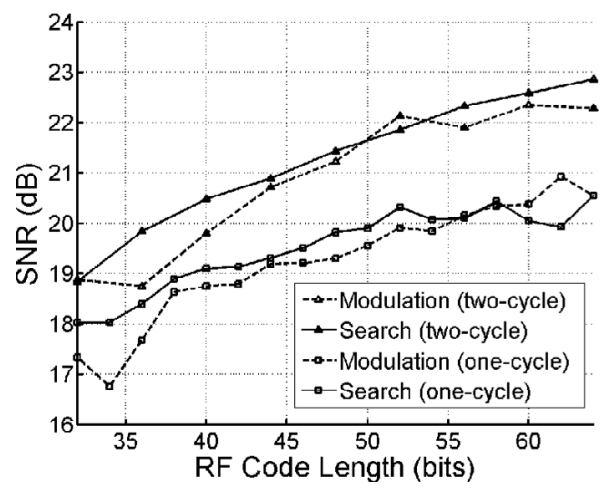

(c)

Fig. 7. Results for the measured transducer response shown in Fig. 6. (a) The efficiencies of codes as functions of their lengths. (b) The filter lengths that resulted in the maximal $E_{\text {cf }}$ values equal to the code efficiencies. (c) The output SNRs corresponding to the filter lengths shown in (b).

$N=32$ to 64 with a step size of 4 using a two-cycle square wave as the base sequence, with the others corresponding to $N=32$ to 64 with a step size of 2 using a one-cycle square wave as the base sequence (Table III). Fig. 7(a) shows the efficiencies of codes as functions of their lengths, and Figs. 7(b) and (c) show the filter lengths that resulted in the maximal $E_{\mathrm{cf}}$ values (that equal the code efficiencies) and the corresponding output SNRs, respectively. These results are consistent with those given in Section IV-A. Each code designed using the search approach has a higher efficiency than its corresponding code designed using the modulation approach with the same base sequence, with the difference ranging between 0.5 and $5 \mathrm{~dB}$. The higher 


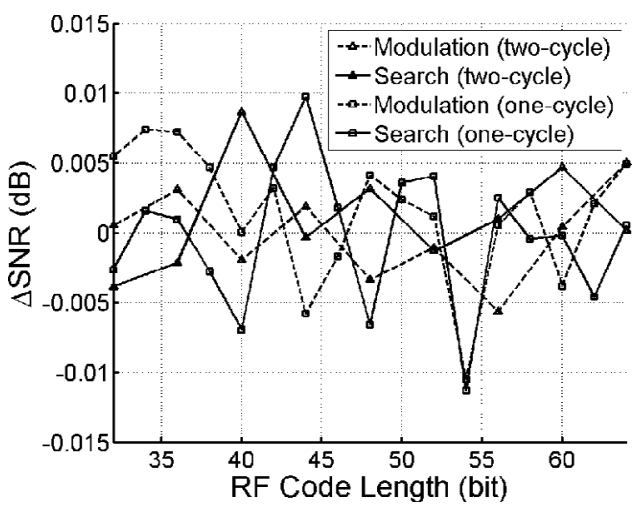

(a)

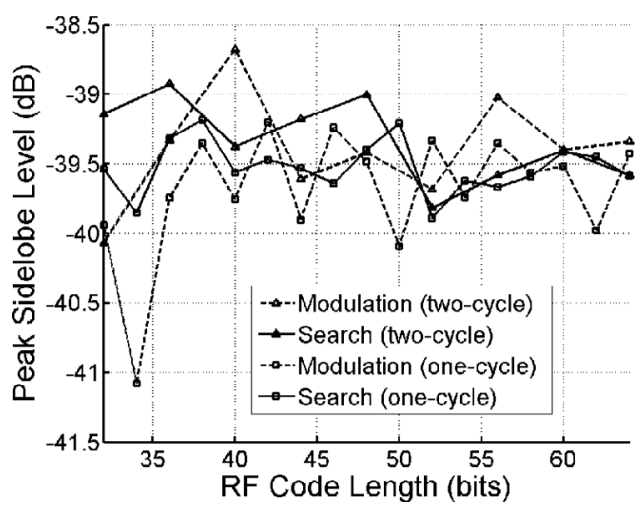

(c)

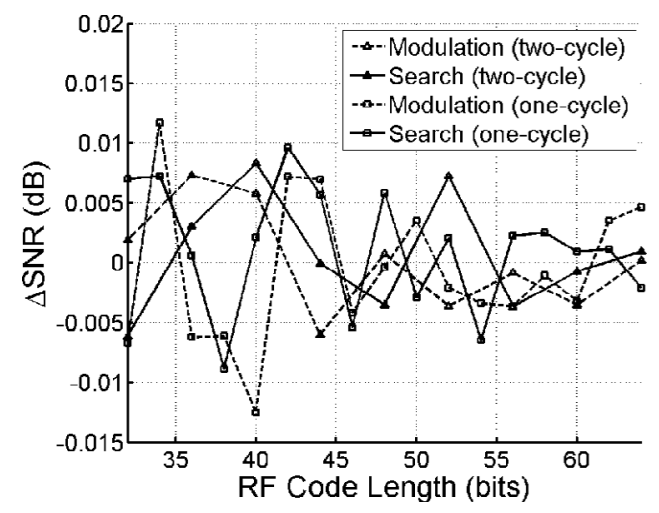

(b)

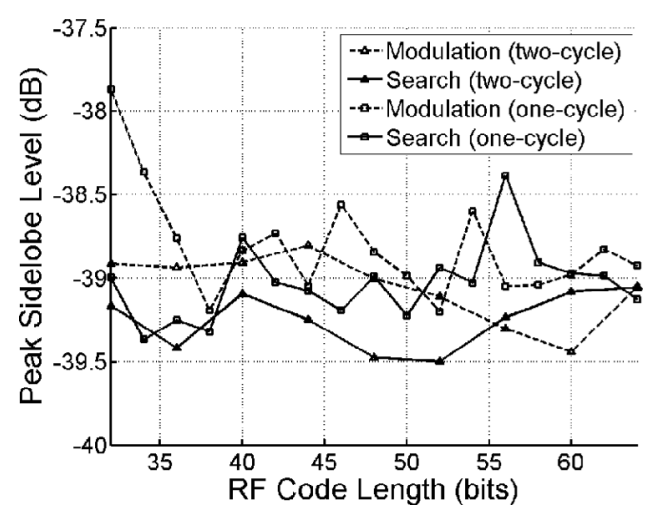

(d)

Fig. 8. Effects of amplitude quantization. (a) $\triangle$ SNRs (differences between SNR improvements with and without amplitude quantization) for the Gaussian-shaped transducer response. (b) $\Delta$ SNRs for the measured transducer response. (c) The peak sidelobe levels for the Gaussianshaped transducer response. (d) The peak sidelobe levels for the measured transducer response.

TABLE III

Hexadecimal Representations of the RF Codes Designed Using the Search Approach for $b(n)=(-1)^{n}$ AND $N_{\mathrm{B}}=4$ (Second Column), And for $b(n)=(-1)^{n}$ And $N_{\mathrm{B}}=2$ (Third Column.$^{1}$

\begin{tabular}{ccc}
\hline & \multicolumn{2}{c}{ Search approach } \\
\cline { 2 - 3 }$N$ & $N_{\mathrm{B}}=4$ & $N_{\mathrm{B}}=2$ \\
\hline 32 & $252 \mathrm{~A} 5551$ & 0C96B557 \\
34 & & 03AAB5A4C \\
36 & 2D4A85551 & 7A52AAC93 \\
38 & & 096D2A55DD \\
40 & $5555 \mathrm{AB} 52 \mathrm{DB}$ & 3F555AD6C9 \\
42 & & 0DD55EA52DB \\
44 & $55552 \mathrm{AD} 4 \mathrm{~B} 09$ & 22AAD4A436C \\
46 & & 027B5AB5574C \\
48 & 692952A15555 & 2484D554AD78 \\
50 & & 02254AA2A7B49 \\
52 & 696B56AB55551 & 32E8AA950B4B4 \\
54 & & 18F5A9555B693F \\
56 & 755556AB54A5AD & 09252B52AABB67 \\
58 & & 092127AAAB56B76 \\
60 & 155556AB54AD2D6 & 212D4ABD4514D9F \\
62 & & 1B68FB5556B57A73 \\
64 & 0B5A56A955AAAAA8 & 0745145AAD4B4A67 \\
\hline
\end{tabular}

${ }^{1}$ The transducer response measured with the setup shown in Fig. 5 is shown in Fig. 6, and the additive noise sampled by the ADC was assumed to be white. code efficiency for the search approach relative to the modulation approach is mainly attributable to the reduction in filter length. With the two- and one-cycle base sequences, the ratios of the filter length to the code length range over $[1.4,2.3]$ and $[1.5,2.2]$ for the search approach, respectively, and over $[2.9,7.4]$ and $[2.4,4.8]$ for the modulation approach. Note that, in general, the code efficiencies are lower than those obtained in Section IV-A. This is due to the definition of SNR reference given at the beginning of this section. Because the transducer considered in the subsection has a wider bandwidth, a higher echo signal level will be obtained when a single-bit signal is transmitted. Therefore, setting the SNR corresponding to a single-bit firing signal to 1 equivalently assumes more noise. Also note that the codes designed using the modulation approach with the one-cycle base sequence adapt to the increased transducer bandwidth better than the other three groups of codes. Nonetheless, in terms of improving the SNR, those codes are worse than the others.

\section{Quantization Effects}

All the above results were obtained based on floatingpoint operations. In practical situations, filter coefficients may be quantized to fixed-point numbers. To evaluate the effects of amplitude quantization on pulse compression performance, for each code we quantized the real 
and imaginary parts of the compression filter $h_{\mathrm{o}}(n)$ to 8 bits, repeated the pulse compression procedure, and compared the resultant $\mathrm{SNR}, \mathrm{SNR}_{\mathrm{dB}, \mathrm{q}}$, with the $\mathrm{SNR}$ corresponding to the ideal filter, $\mathrm{SNR}_{\mathrm{dB}, \mathrm{i}}$. Define $\Delta \mathrm{SNR}$ as $\mathrm{SNR}_{\mathrm{dB}, \mathrm{q}}-\mathrm{SNR}_{\mathrm{dB}, \mathrm{i}}$. Figs. 8(a) and (b) show the $\Delta$ SNRs as functions of the code length for the Gaussian-shaped and measured transducer responses, respectively. All the $\triangle \mathrm{SNR}$ values are small. Note that $\triangle \mathrm{SNR}$ is equal to the difference between code efficiencies with and without amplitude quantization. Figs. 8(c) and (d) shows the peak sidelobe levels for the Gaussian-shaped and measured transducer responses, respectively. The results show that amplitude quantization only slightly degraded the pulse compression performance.

\section{Conclusions}

This paper proposes an approach to designing binary codes having a low sampling ratio and providing efficient SNR improvements. Unlike the conventional modulation approach in which a base sequence is used to modulate wideband sequences up to the transducer passband, which thereby excludes a major portion of the codes as possible RF codes, the proposed search approach uses a genetic algorithm to search all the codes that match the transducer passband; hence, the resultant codes can improve the SNR more efficiently. In the study, the bit rate was set to $50 \mathrm{MHz}$ in order to keep the sampling ratios about 2 , and two transducer responses were considered in order to demonstrate the adaptability of the proposed approach. Assuming that the transducer has an ideal Gaussian frequency response with a center frequency of $25 \mathrm{MHz}$ and a $-6 \mathrm{~dB}$ bandwidth of $15 \mathrm{MHz}$, the codes designed using the proposed approach outperformed those designed using the modulation approach by 1 to $6 \mathrm{~dB}$ in terms of code efficiency (i.e., in terms of the capacity for improving the SNR normalized by the total length of the code and the filter). When a real transducer with a center frequency of $26.4 \mathrm{MHz}$ and a one-way $-6 \mathrm{~dB}$ bandwidth of $20.7 \mathrm{MHz}$ was considered, the codes designed using the proposed approach were superior by 0.5 to $5 \mathrm{~dB}$. Our approach can be applied to designing binary codes with SNR improvements in high-frequency ultrasound systems, for which using arbitrary-waveform transmitters may be infeasible. A reasonable bit rate of $50 \mathrm{MHz}$ will suffice if the ultrasonic center frequency is $25 \mathrm{MHz}$.

\section{ACKNOWLEDGMENTS}

The authors would like to thank Dr. Matt O'Donnell for providing the equipment, including the pulser/receiver and the oscilloscope, necessary for measuring the transducer impulse response, and the reviewers for numerous helpful comments.

\section{REFERENCES}

[1] M. O'Donnell, "Coded excitation system for improving the penetration of real-time phased-array imaging systems," IEEE
Trans. Ultrason., Ferroelect., Freq. Contr., vol. 39, no. 3, pp. 341-351, 1992.

[2] R. Y. Chiao and X. Hao, "Coded excitation for diagnostic ultrasound: A system developer's perspective," IEEE Trans. Ultrason., Ferroelect., Freq. Contr., vol. 52, no. 2, pp. 160-170, 2005.

[3] M. O'Donnell and Y. Wang, "Coded excitation for synthetic aperture ultrasound imaging," IEEE Trans. Ultrason., Ferroelect., Freq. Contr., vol. 52, no. 2, pp. 171-176, 2005.

[4] Y. Wang, K. Metzger, D. N. Stephens, G. Williams, S. Brownlie, and M. O'Donnell, "Coded excitation with spectrum inversion (CEXSI) for ultrasound array imaging," IEEE Trans. Ultrason., Ferroelect., Freq. Contr., vol. 50, no. 7, pp. 805-823, 2003.

[5] R. L. Haupt and S. E. Haupt, Practical Genetic Algorithms. New York: Wiley, 1998.

[6] S.-W. Huang and P.-C. Li, "Arbitrary waveform coded excitation using bipolar square wave pulsers in medical ultrasound," IEEE Trans. Ultrason., Ferroelect., Freq. Contr., vol. 53, no. 1, pp. 106-116, 2006.

[7] B. Haider, P. A. Lewin, and K. E. Thomenius, "Pulse elongation and deconvolution filtering for medical ultrasonic imaging," IEEE Trans. Ultrason., Ferroelect., Freq. Contr., vol. 45, no. 1 , pp. 98-113, 1998.

[8] A. V. Oppenheim and R. W. Schafer, Discrete-Time Signal Processing. Englewood Cliffs, NJ: Prentice-Hall, 1989.

[9] J. Ruprecht and M. Rupf, "On the search for good aperiodic binary invertible sequences," IEEE Trans. Inform. Theory, vol. 42, no. 5, pp. 1604-1612, 1996.

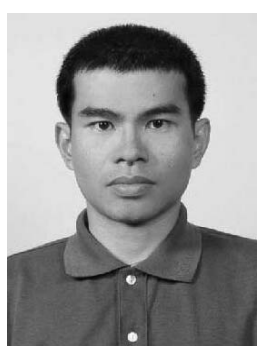

Sheng-Wen Huang was born in 1971 in Changhua, Taiwan, R.O.C. He received the B.S. and Ph.D. degrees from National Taiwan University, Taipei, Taiwan, R.O.C., in 1993 and 2004, respectively, both in electrical engineering. He worked as a postdoctoral researcher at National Taiwan University from 2004 to 2005 , and is currently a postdoctoral researcher with the Department of Biomedical Engineering at the University of Michigan. His current research interests include thermal strain imaging, coded excitation, and optoacoustic imaging.

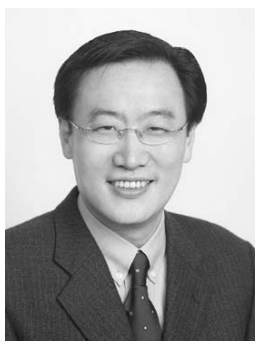

Pai-Chi Li (S'93-M'95-SM'01) received the B.S. degree in electrical engineering from National Taiwan University, Taipei, Taiwan, R.O.C., in 1987, and the M.S. and Ph.D. degrees from the University of Michigan, Ann Arbor, MI, in 1990 and 1994, respectively, both in electrical engineering: systems.

He was a research assistant with the Department of Electrical Engineering and Computer Science of the University of Michigan from 1990 to 1994. He joined Acuson Corporation, Mountain View, CA, as a member of the Technical Staff in June 1994. His work with Acuson was primarily in the areas of medical ultrasonic imaging system design for both cardiology and general imaging applications. In August 1997, he went back to the Department of Electrical Engineering at National Taiwan University, where he is currently Professor of Department of Electrical Engineering and Director of Institute of Biomedical Electronics and Bioinformatics. His current research interests include biomedical ultrasonic imaging and signal processing.

Dr. Li is a senior member of IEEE and Associate Editor of IEEE Transactions on Ultrasonics, Ferroelectrics, and Frequency Control. He is also on the Editorial Board of Ultrasonic Imaging. He received the 2005 Outstanding Electrical Engineering Professor Award, the Chinese Institute of Electrical Engineering, the 2004 Distinguished Research Award, National Science Council, the 2004 Distinguished Research Achievement Award, National Taiwan University, the 2003 Outstanding Researcher Award, National Taiwan University, the 
2002 Dr. Wu Dayou Research Award from National Science Council, the 2002 Outstanding Young Electrical Engineer Award from Chinese Institute of Electrical Engineering and the Distinguished Industrial Collaboration Award of Ministry of Education. He was also the recipient of the Distinguished Achievement Award in Electrical Engineering: Systems in 1994 for his outstanding academic achievement at the University of Michigan. 